\title{
EUS-guided sampling with 25G biopsy needle as a rescue strategy for diagnosis of small subepithelial lesions of the upper gastrointestinal tract
}

\section{(c) $(>)$}

\author{
Authors \\ Filippo Antonini ${ }^{1}$, Sara Giorgini ${ }^{2}$, Lorenzo Fuccio ${ }^{3}$, Lucia Angelelli ${ }^{4}$, Giampiero Macarri ${ }^{1}$
}

Institutions

1 Department of Gastroenterology, A. Murri Hospital, Polytechnic University of Marche, Fermo, Italy

2 Pathological Anatomy and Histopathology, Department of Biomedical Sciences and Public Health, Polytechnic University of Marche, Ancona, Italy

3 Department of Medical and Surgical Sciences, S. OrsolaMalpighi Hospital, University of Bologna, Bologna, Italy

4 Medical Oncology, Mazzoni Hospital, Ascoli Piceno, Italy

submitted 6.1.2018

accepted after revision 13.3.2018

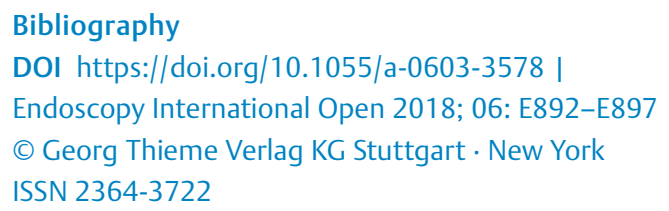

Corresponding author

Filippo Antonini, MD, UOC Gastroenterologia ed

Endoscopia Digestiva, Università Politecnica delle Marche, Ospedale "A.Murri”, 63900 - Fermo, Italy

Fax: +39.0734 .6252252$

filippore@yahoo.it

\section{ABSTRACT}

Background and study aims This study was designed to evaluate the impact of additional tissue obtained with endoscopic ultrasound (EUS)-guided 25-gauge core biopsy needle (25G-PC) following an unsuccessful fine-needle biopsy (FNB) performed with larger-bore needles for the characterization of gastrointestinal subepithelial lesions (GI-SELs).

Patients and methods We prospectively collected and retrospectively analyzed information in our database from January 2013 to June 2017 for all patients with GI-SELs who received a EUS-guided FNB (EUS-FNB) with 25G-PC during the same procedure after failure of biopsy performed with larger-bore needle. Diagnostic yield, diagnostic accuracy and procedural complications were evaluated.

Results Sixteen patients were included in this study, 10 men and 6 women, median age 67.8 (range 43 to 76 years). Five patients were found to have a SEL localized in the distal duodenum, five in the gastric antrum, two in the gastric fundus and four in the gastric body. The mean size of the lesions was $20.5 \mathrm{~mm}$ (range $18-24 \mathrm{~mm}$ ). EUS-FNB with 25GPC enabled final diagnosis in nine patients (56.2\%). Regarding the subgroup of duodenal lesions, the procedure was successful in four of five (80\%). Final diagnoses with EUSguided sampling were GIST $(n=6)$, leiomyoma $(n=2)$ and metastatic ovarian carcinoma $(n=1)$. No procedure-related complications were recorded.

Conclusion In patients with small GI-SELs, additional tissue obtained with 25G-PC could represents a "rescue" strategy after an unsuccessful procedure with larger-bore needles, especially when lesions are localized in the distal duodenum.

\section{Introduction}

Gastrointestinal subepithelial lesions (SELs) include several neoplastic and non-neoplastic lesions that can be difficult to diagnose. The term "subepithelial" seems more appropriate than "submucosal" because these lesions can originate from each layer of the gastrointestinal wall or even can be caused by extramural compression [1]. Most of the gastrointestinal SELs are asymptomatic, therefore their real incidence is unknown. The highest incident of SELs throughout the gastrointestinal tract has been documented in the stomach [2]. When a SEL is found, establishing the exact nature is mandatory for subsequent management. Conventional endoscopic biopsies are frequently inconclusive, because mucosa overlying SELs is usually normal. Similarly, radiological investigations such as barium contrast radiography, computed tomography (CT) or abdominal ultrasound are of limited value in defining the exact nature of the lesion $[1,3]$.

Endoscopic ultrasound (EUS) is currently recommended as first-choice investigation to assess SELs because of its accuracy 
in differentiating them from extrinsic compression and providing information about morphology and layer of origin [1]. EUS can sometimes provide information in case of lesions with typical morphological features, such as lipomas or duplication cysts. However, tissue diagnosis is often required, especially in neoplasms for which immunohistochemistry (IHC) is mandatory, such as gastrointestinal stromal tumors (GISTS). EUS needles of different size and shape have been used, with variable success/complication rates [4-7]. Recently, a new needle with reverse bevel technology has been developed to simultaneously obtain cytological aspirates and histological core samples, thereby leading to an ideal EUS-guided fine needle biopsy (EUS-FNB) [8-11]. The majority of reports on EUS-FNB needles have focused on pancreatic masses. Data on the diagnostic performance of 25-gauge $(G)$ core needle to assess GI-SELs are lacking.

The aim of this study was to evaluate the impact of additional tissue obtained with EUS-guided 25G-needle core biopsy following an inconclusive EUS-FNB performed with larger-bore needles for characterization of GI-SELs.

\section{Patients and methods}

\section{Patients}

All consecutive patients who received, during the same procedure, an EUS-guided FNB with 25G-ProCore (25G-PC) needle (EchoTip ProCore; Cook Endoscopy) as a "rescue strategy" after an initial unsuccessful biopsy performed with larger ProCore needles (22G-PC and/or 19G-PC) to diagnose upper GI-SELs were prospectively enrolled and retrospectively analyzed. EUSFNB with 25G-PC was considered as a rescue strategy after a prior attempt with EUS-FNB with a larger-bore needle when: (1) puncture of the lesion was not feasible for technical reasons (i.e, difficulty to advance the needle through the scope in angulated position); or (2) specimens obtained were considered macroscopically suboptimal (i.e, not suitable to put in a formalin bottle for histological examination).

Inclusion criteria for EUS-FNB were: (1) presence of upper GISELs revealed by endoscopy, (2) need for pathological assessment to make a diagnosis and/or to guide management decision, (3) age older than 18 years, and (4) ability to provide informed consent. Exclusion criteria were: (1) inability to provide informed consent; (2) evidence of coagulation disorder.

Baseline variables are presented as numbers (percentage) and mean values (range).

\section{EUS-FNB procedure}

EUS-FNBs were performed by using convex array echoendoscopes (UCT-140, Olympus America, Inc. Melville, New York, United States) with the patient in the left lateral position under conscious sedation (intravenous fentanyl and midazolam) or deep sedation (propofol). After targeting the optimal puncture site, each puncture was done using a core biopsy needle (EchoTip ProCore; Cook Endoscopy) guided by real-time EUS imaging. Two different suction techniques (slow-pull and "wet") were used at the operator's discretion. In the slow-pull technique, the stylet was left inside the needle and, after punctur- ing the lesion, it was slowly and continuously removed as the needle was moved to-and-fro for 10 to 15 times inside the lesion. In the "wet" technique, the stylet was removed and the needle was filled with saline to replace the column of air with water, then the needle was passed into the lesion and the suction applied with a 10-cc pre-vacuum syringe. Thereafter, the needle was moved to-and-fro 10 to 15 times inside the lesion, syringe-suction was then turned off before withdrawing the needle from the lesion $[12,13]$. The ProCore (PC) needle size for the first attempt (19G or 22G) and the number of needle passes were at discretion of the endosonographer. The procedure was stopped when biopsy specimens were considered sufficient by the operator at gross examination. A maximum number of three biopsy attempts were allowed for each needle.

All EUS-FNBs procedures were performed by a single experienced endoscopist (FA) who has performed more than 1000 EUS procedures and at least 100 EUS-FNAs per year. This study was approved by the institutional review board.

\section{Specimen evaluation and histological process}

During the procedure there was no on-site cytopathologist. After EUS-FNB, the sample obtained was expelled onto slides. All macroscopically visible cores specimens (defined as whitish or yellowish piece of tissue with an apparent bulk) considered adequate by the endosonographer were put into formalin for histological process. Specimens considered inadequate were submitted for cytology assessment.

Histologic specimen was categorized "diagnostic" when considered adequate to reach a definitive diagnosis by the pathologist (including cases where IHC was mandatory), and "non-diagnostic" when the sample did not meet this requirement. IHC staining was performed using commercially available antibodies against c-kit (CD117), CD34, S-100, DOG-1, and smooth-muscle actin.

\section{Endpoints}

The primary endpoint was adequacy, defined as the rate of cases in which an adequate tissue specimen for histological examination was obtained.

Secondary endpoints were accuracy, defined as proportion of correct diagnoses, and adverse event rate. Standard references for the diagnosis were the surgical specimen when available or other diagnostic investigations and a follow-up of at least 6 months. Early (within 48 hours) and late (> 48 hours) adverse events (AEs) were recorded.

All patients were evaluated for procedural AEs with a phone call or clinic visit at 24 to 48 hours and at 7 to 10 days following the procedure.

\section{Results}

Between January 2013 and June 2017, a total of 108 patients were referred to our department for tissue sampling of upper GI-SELs. Among them, 16 (14.8\%) patients (10 male; median age, 67.8 years; range, 43 to 76 years) underwent EUS-FNB with 25G-PC as a rescue strategy after an initial inconclusive biopsy performed with larger-bore needles during the same EUS procedure ( $\triangleright$ Table 1$)$. Five patients had a SEL localized in 
- Table 1 Patient characteristics in 16 cases of upper GI-SELs.

\begin{tabular}{|l|l|}
\hline Patient characteristics & 16 cases of GI-SELs \\
\hline Mean age (years, range) & $67.8(43-76)$ \\
\hline Male: female & $10: 6$ \\
\hline Tumor location, $\mathrm{n}(\%)$ & \\
\hline - gastric fundus & $2(12.5)$ \\
\hline - gastric body & $4(25)$ \\
\hline - gastric antrum & $5(31.2)$ \\
\hline - duodenum & $5(31.2)$ \\
\hline Tumor size (mm, range) & $20.5(18-24)$ \\
\hline Layer of origin on EUS, n (\%) & $16(100)$ \\
\hline - Fourth & \\
\hline $\begin{array}{l}\text { GI-SEL, gastrointestinal subepithelial lesions; EUS, endoscopic ultrasonogra- } \\
\text { phy }\end{array}$ & \\
\hline
\end{tabular}

the distal duodenum, five in the antrum, two in the gastric fundus and four in the gastric body. All SELs originated from the fourth sonographic layer of the gastrointestinal wall (i.e, muscularis propria) and showed a homogeneous hypoechoic echo pattern on EUS. Mean size of the lesions was $20.5 \mathrm{~mm}$ (range 18-24mm). Previous EUS-FNB with larger size-needle (11 cases with 22G-PC needle and 5 cases with 19G-PC needle) failed in 10 cases because macroscopically suboptimal specimens were retrieved and in the other six cases because of technical issues ( $\triangleright$ Table 2 ). Technical failure was mainly due to difficulty in advancing a large needle through the scope in an angulated position (such as the distal duodenum) and for the tendency of the needle to push the scope away from the gastrointestinal wall (as it happens in the greater curvature of the stomach).

EUS-FNB with 25G-PC was technically feasible in all subjects and enabled final diagnosis in nine out of 16 cases (56.2\%). IHC was feasible in all these adequate specimens. Regarding the subgroup of duodenal lesions, the procedure was successful in four of five $(80 \%)$ ( $\triangleright$ Fig. 1). Final diagnoses with EUS-guided sampling were GIST $(n=6)$, leiomyoma $(n=2)$ and metastatic ovarian carcinoma $(n=1)$. All six patients with EUS-proven GIST were treated by surgery and confirmed at final pathology. Patients with leiomyoma were planned for follow-up. The patient with metastasis from ovarian cancer started palliative chemotherapy. Regarding the seven patients with non-diagnostic results with 25G-PC, two underwent wedge resection and GIST was confirmed on surgical specimens in both cases, five had endoscopic follow-up (no change was seen in a mean followup period of 23 months, ranging from 7 to 38 months).

No major procedure-related AEs were recorded irrespective of needle size.

- Table 2 Technical results of EUS-guided fine-needle biopsy.

\begin{tabular}{|c|c|c|c|c|c|}
\hline $\begin{array}{l}\text { Tumor } \\
\text { location }\end{array}$ & $\begin{array}{l}\text { Tumor size } \\
\text { on EUS }\end{array}$ & $\begin{array}{l}\text { Needle size at the first } \\
\text { attempt (Gauge) }\end{array}$ & $\begin{array}{l}\text { EUS-FNB results of } \\
\text { first attempt }\end{array}$ & $\begin{array}{l}\text { Histological results with } \\
\text { 25-Gauge needle }\end{array}$ & Follow-up \\
\hline $\mathrm{F}$ & 19 & 22 & Failure & Nondiagnostic & Follow-up \\
\hline $\mathrm{F}$ & 22 & 22 & Suboptimal & Nondiagnostic & Follow-up \\
\hline B & 18 & 22 & Suboptimal & Leiomyoma & Follow-up \\
\hline B & 19 & 19 & Failure & Nondiagnostic & Follow-up \\
\hline B & 20 & 22 & Suboptimal & Leiomyoma & Follow-up \\
\hline B & 22 & 19 & Suboptimal & Nondiagnostic & Surgery \\
\hline A & 18 & 22 & Suboptimal & Nondiagnostic & Follow-up \\
\hline A & 20 & 22 & Suboptimal & GIST & Surgery \\
\hline A & 20 & 22 & Suboptimal & GIST & Surgery \\
\hline A & 21 & 22 & Failure & Nondiagnostic & Follow-up \\
\hline A & 23 & 19 & Failure & Metastatic cancer & Chemotherapy \\
\hline D & 19 & 22 & Failure & GIST & Surgery \\
\hline D & 20 & 22 & Suboptimal & GIST & Surgery \\
\hline D & 20 & 19 & Suboptimal & GIST & Surgery \\
\hline D & 23 & 19 & Failure & GIST & Surgery \\
\hline D & 24 & 22 & Suboptimal & Nondiagnostic & Surgery \\
\hline
\end{tabular}



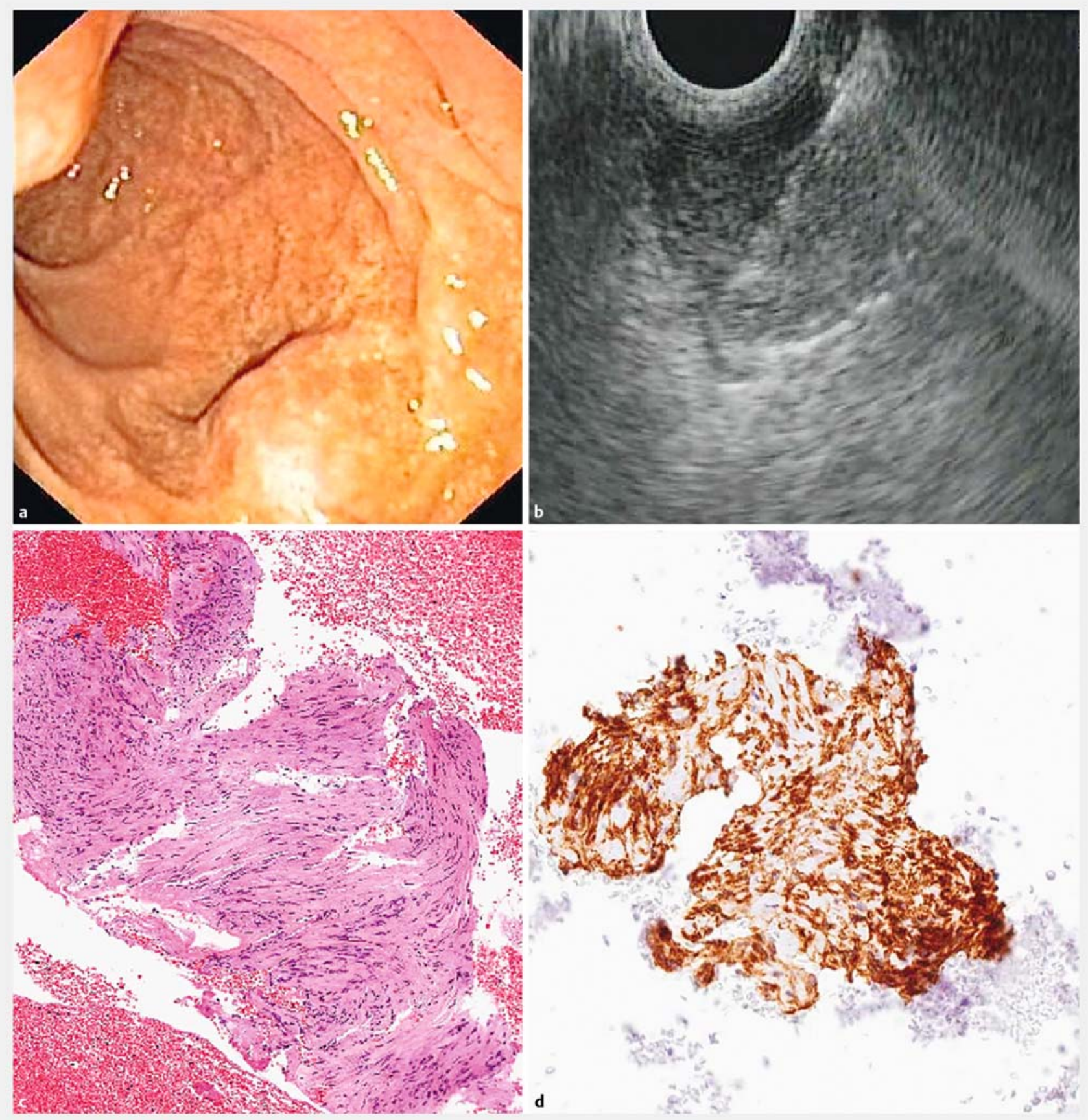

- Fig. 1 Images of a small duodenal GIST. a Endoscopic image of a small subepithelial tumor in the second portion of the duodenum opposite to the ampulla of Vater. $\mathbf{b}$ EUS-guided fine-needle biopsy of the lesion with a 25 -Gauge ProCore needle. The needle can be visualized. $\mathbf{c}$ Histologic examination showing groups of spindled-shaped cells (H\&E staining, $\times 20$ magnification). $\mathbf{d}$ Immunohistochemistry positive for DOG-1 (×20 magnification).

\section{Discussion}

Endoscopic ultrasound (EUS) is considered the primary modality for evaluation of SELs. Furthermore, EUS- FNA enables tissue acquisition when needed. EUS-FNA has an overall diagnostic accuracy ranging from $60 \%$ to $80 \%$ in SELs $[14,15]$. Several factors have been associated with inadequate tissue yield but the main ones are size and location of the lesion [16]. In fact, sam- pling adequacy increases proportionate with tumor size and poorer diagnostic yield has been generally associated with lesions smaller than 30 to $40 \mathrm{~mm}$. Evidence from the literature supports this statement. In a retrospective study by Hoda et al on 112 upper GI-SELs, the diagnostic yield was $44.4 \%$ for lesions less than $10 \mathrm{~mm}$ and increased up to $58.3 \%$ for lesions ranging from 11 to $30 \mathrm{~mm}$, and to $69.7 \%$ for lesions > $30 \mathrm{~mm}$ [14]. In another study on 53 subepithelial gastric lesions, EUS-FNA had an 
overall diagnostic yield of $71 \%$ for lesions measuring up to 20 $\mathrm{mm}, 86 \%$ for lesions ranging 20 to $40 \mathrm{~mm}$ and $100 \%$ for lesions larger than $40 \mathrm{~mm}$ [17]. More recently Akahoshi's group obtained a diagnostic rate of $73 \%$ from EUS-FNA of 90 gastric SELs smaller than $20 \mathrm{~mm}$ [18]. However, Sekine et al demonstrated that GIST can be correctly identified by EUS-FNA even in small lesions, with an overall sensitivity of $82.5 \%$ for GIST of any size, and $81.3 \%$ for GIST smaller than $20 \mathrm{~mm}$ [19].

Unfortunately, cytology is often not sufficient to reach a definitive diagnosis of GI-SELs and usually a proper histological sample is required, especially in view of IHC analysis. EUS-FNB PC needles have been conceived to obtain more tissue and ideally to provide histological specimen (core biopsy). Studies on core biopsy needles were mainly conducted on patients with pancreatic masses, while only a few studies are available looking at characterization of SELs [7,20-22]. In the first experience of Iglesias-Garcia et al on heterogeneous study population with intestinal and extra-intestinal lesions, EUS-FNB with 19GPC was technically feasible in $98.2 \%$ of cases $(112 / 114)$. In this study, 11 patients presented with upper GI-SELs and correct diagnoses were achieved in nine of them (81.8\%) [8]. Kim et al have evaluated 12 patients with upper SELs, including esophageal, gastric and duodenal lesions, and EUS-FNB with a 22G-PC needle reached a diagnostic yield of $75 \%$ [20]. Similarly, Lee et al evaluated the efficacy of EUS-FNB with 22G-PC needle in gastric SEL, obtaining an overall diagnostic yield of $86 \%$ [21]. According to tumor location, the highest diagnostic yield was in the fundus (100\%), followed by the body (89.5\%), cardia (83.3\%), and antrum (50\%). In this study there were only two cases of antral lesions and only one had final diagnosis with EUS-FNB [21]. More recently, a larger study of 77 upper GISELs with EUS biopsy needle has been conducted to evaluate performance of EUS-FNB using a 22G-PC where diagnosis was achieved in $81.8 \%$ of cases [22]. Core biopsy tissue was obtained in $96.8 \%$ of the cases. Only a single case of post-procedural bleeding was recorded [22]. Recently, a new 20G-PC needle has been developed, which is expected to be a balanced compromise between flexibility, facility of use proper of the smallest needles, and quality of the tissue sampling, typical of the larger needle, providing echo endoscopists a new tool to accurately target lesions, regardless of their size or location [7]. Antonini et al published the first experience with this needle in a multicenter retrospective study for the diagnosis of SELs. A total of 50 SELs were included and after a mean number of passes of 2.2 (range $1-4$ ), definitive diagnosis with full histological assessment including IHC was obtained in $88 \%$ of patients (44/ 50) without any major complications [7].

The external validity of these studies was strongly limited by the fact that most of the punctured lesions were $>20 \mathrm{~mm}$ in $\mathrm{di}$ ameter and a 22G-PC needle was used. Notably, in the current study, all the lesions were sampled with a 25G-PC needle and all of them were less than $25 \mathrm{~mm}$. Indeed, our study showed than even in lesions $\leq 20 \mathrm{~mm}$, the 25G-PC was able to achieve a diagnosis in $70 \%$ of cases ( $7 / 10)$.

Up to now, management algorithms for small GIST have been a matter for debate $[23,24]$. Natural history of small GISTs has not been well defined but even these lesions may present with malignant behavior and evolve into clinically relevant lesions $[25,26]$. Therefore, the European Society for Medical Oncology (ESMO) recommends EUS assessment for esophagogastric or duodenal SELs $<20 \mathrm{~mm}$ and surgical excision of histologically proven small GISTs, unless that entails major morbidity [27].

EUS-guided tissue acquisition with 25G-PC needles in patients with pancreatic lesions resulted in high diagnostic yield, similar to standard 25-gauge FNA needles, able also to provide sufficient tissue specimen for histological assessment $[28,29]$. In the study by Iwashita et al, despite the low yield (32\%) of a real "core," histological analysis was possible in $63 \%$ of patients on the first pass and in $80 \%$ of cases on subsequent passes [28]. This indicated that a definitive diagnosis could be obtained based also on tissue fragments that do not meet the criteria for architecturally intact histology but can still yield a diagnosis based on cell morphology. In our study both histological core and tissue fragments were considered by the pathologist for the final diagnosis, including full $\mathrm{IHC}$ when required. The results show that EUS-FNB with 25G-PC enables definitive diagnosis in most of the assessed small upper GI-SELs, otherwise not fully characterized by other larger-bore needles. Indeed, correct diagnosis rates were $56.2 \%$ overall but $80 \%$ in duodenal lesions.

Other authors have highlighted the better performance of the $25 \mathrm{G}$ needle for SELs located in certain positions, such as the greater curvature of the stomach, where the needle tip may rebound, making it difficult to puncture the lesion [19]. The major advantage of the $25 \mathrm{G}$ needle is its thin caliber which makes EUS-guided sampling easier even in difficult sites. Transduodenal EUS-guided tissue acquisition can be technically challenging due to the angulated position that may hamper advancement of the needle through the scope and into the targeted lesion. Moreover, to avoid instrumental damage with larger-bore needles, often the scope has to be withdrawn into the stomach so the tip can be straightened.

Our study presents some limitations that should be acknowledged. The number of patients was relatively small and there were recruited in a single center (reducing the external validity of our findings). Follow-up was relatively short, ranging from 7 to 38 months after EUS-FNB, and not all patients underwent surgical resection as the gold standard for diagnosis. Followup of small GI-SELs is controversial. Koizumi et al. have showed that doubling time differs according to the type of SELs, and GISTs were confirmed to have a significantly shorter doubling time (17.2 months) than the other types of tumors, thus suggesting that even small SELs should initially be followed up within at least 6 months after detection [30].

To the best of our knowledge, this study represents the first investigation of the role of EUS-FNB with 25G-PC biopsy following a failed FNB performed with another size needle for characterization of small subepithelial lesions of the upper gastrointestinal tract. Therefore, larger prospective studies are warranted to confirm our results. 


\section{Conclusion}

In conclusion, our study shows that in patients with small GISELs, additional tissue obtained with 25G-PC may represents a "rescue" strategy after an unsuccessful procedure with largerbore needles, especially when lesions are localized in the distal duodenum.

\section{Competing interests}

None

\section{References}

[1] Landi B, Palazzo L. The role of endosonography in submucosal tumours. Best Pract Res Clin Gastroenterol 2009; 23: 679-701

[2] Papanikolaou IS, Triantafyllou K, Kourikou A et al. Endoscopic ultrasonography for gastric submucosal lesions. World J Gastrointest Endosc 2011; 3: 86-94

[3] Ponsaing LG, Kiss K, Loft A et al. Diagnostic procedures for submucosal tumors in the gastrointestinal tract. World J Gastroenterol 2007; 13: $3301-3310$

[4] Binmoeller KF, Thul R, Rathod V et al. Endoscopic ultrasound-guided, 18-gauge, fine needle aspiration biopsy of the pancreas using a 2.8 mm channel convex array echoendoscope. Gastrointest Endosc 1998; 47: $335-340$

[5] Wiersema M], Levy M], Harewood GC et al. Initial experience with EUS-guided Trucut needle biopsy of perigastric organs. Gastrointest Endosc 2002; 56: 275 - 278

[6] Levy M], Jondal ML, Clain J et al. Preliminary experience with an EUSguided trucut biopsy needle compared with EUS-guided FNA. Gastrointest Endosc 2003; 57: 101 - 106

[7] Antonini F, Delconte G, Fuccio L et al. Endoscopic ultrasound-guided tissue sampling with a 20-gauge core biopsy needle for the characterization of gastrointestinal subepithelial lesions: a multicenter study. Endosc Ultrasound 2018: doi:10.4103/eus.eus_95_17

[8] Iglesias-Garcia J, Poley JW, Larghi A et al. Feasibility and yield of a new EUS histology needle: results from a multicenter, pooled, cohort study. Gastrointest Endosc 2011; 73: 1189-1196

[9] Larghi A, Iglesias-Garcia J, Poley JW et al. Feasibility and yield of a novel 22-gauge histology EUS needle in patients with pancreatic masses: a multicenter prospective cohort study. Surg Endosc 2013; 27: $3733-3738$

[10] Fabbri C, Luigiano C, Maimone A et al. Endoscopic ultrasound-guided fine-needle biopsy of small solid pancreatic lesions using a 22-gauge needle with side fenestration. Surg Endosc 2015; 29: 1586-1590

[11] Antonini F, Fuccio L, Giorgini S et al. Biliary plastic stent does not influence the accuracy of endoscopic ultrasound-guided sampling of pancreatic head masses performed with core biopsy needles. Dig Liver Dis 2017; 49: 898-902

[12] Attam R, Arain MA, Bloechl S] et al. "Wet suction technique (WEST)": a novel way to enhance the quality of EUS-FNA aspirate. Results of a prospective, single-blind, randomized, controlled trial using a 22-gauge needle for EUS-FNA of solid lesions. Gastrointest Endosc 2015; 81: $1401-1407$

[13] Antonini F, Fuccio L, Fabbri C et al. Endoscopic ultrasound-guided tissue acquisition of pancreatic masses with core biopsy needles using wet suction technique. Endosc Ultrasound 2017; 6: 73-74

[14] Hoda KM, Rodriguez SA, Faigel DO. EUS-guided sampling of suspected Gl stromal tumors. Gastrointest Endosc 2009; 69: 1218-1223

[15] Mekky MA, Yamao K, Sawaki A et al. Diagnostic utility of EUS-guided FNA in patients with gastric submucosal tumors. Gastrointest Endosc 2010; 71: 913-919

[16] Suzuki T, Arai M, Matsumura T et al. Factors associated with inadequate tissue yield in EUS-FNA for gastric SMT. ISRN Gastroenterol 2011; 2011: 619128

[17] Akahoshi K, Sumida $\mathrm{Y}$, Matsui $\mathrm{N}$ et al. Preoperative diagnosis of gastrointestinal stromal tumor by endoscopic ultrasound-guided fine needle aspiration. World J Gastroenterol 2007; 13: 2077-2082

[18] Akahoshi K, Oya M, Koga T et al. Clinical usefulness of endoscopic ultrasound-guided fine needle aspiration for gastric subepithelial lesions smaller than $2 \mathrm{~cm}$. J Gastrointestin Liver Dis 2014; 23: 405-412

[19] Sekine M, Imaoka H, Mizuno N et al. Clinical course of gastrointestina stromal tumor diagnosed by endoscopic ultrasound-guided fine-needle aspiration. Dig Endosc 2015; 27: 44- 52

[20] Kim GH, Cho YK, Kim EY. Korean EUS Study Group. et al. Comparison of 22-gauge aspiration needle with 22-gauge biopsy needle in endoscopic ultrasonography-guided subepithelial tumor sampling. Scand J Gastroenterol 2014; 49: 347-354

[21] Lee M, Min BH, Lee $\mathrm{H}$ et al. Feasibility and diagnostic yield of endoscopic ultrasonography-guided fine needle biopsy with a new core biopsy needle device in patients with gastric subepithelial tumors. Medicine (Baltimore) 2015; 94: e1622

[22] Lee JH, Cho C], Park YS et al. EUS-guided 22-gauge fine needle biopsy for the diagnosis of gastric subepithelial tumors larger than $2 \mathrm{~cm}$. Scand J Gastroenterol 2016; 51: 486-493

[23] Akahoshi K, Oya M. Gastrointestinal stromal tumor of the stomach: How to manage? World J Gastrointest Endosc 2010; 2: 271-277

[24] Kida M, Kawaguchi Y, Miyata E et al. Endoscopic ultrasonography diagnosis of subepithelial lesions. Dig Endosc 2017; 29: 431-443

[25] Yegin EG, Duman DG. Small EUS-suspected gastrointestinal stromal tumors of the stomach: An overview for the current state of management. Endosc Ultrasound 2016; 5: 69-77

[26] Aso A, Ihara E, Kubo $\mathrm{H}$ et al. Gastric gastrointestinal stromal tumor smaller than $20 \mathrm{~mm}$ with liver metastasis. Clin J Gastroenterol 2013; 6: $29-32$

[27] ESMO/European Sarcoma Network Working Group. Gastrointestinal stromal tumours: ESMO Clinical Practice Guidelines for diagnosis, treatment and follow-up. Ann Oncol 2014; 25: (Suppl. 03): iii21 - iii6

[28] Iwashita T, Nakai Y, Samarasena JB et al. High single-pass diagnostic yield of a new 25-gauge core biopsy needle for EUS-guided FNA biopsy in solid pancreatic lesions. Gastrointest Endosc 2013; 77: 909-915

[29] Attili F, Petrone G, Abdulkader I et al. Accuracy and inter-observer agreement of the Procore ${ }^{\mathrm{TM}} 25$ gauge needle for endoscopic ultrasound-guided tissue core biopsy. Dig Liver Dis 2015; 47: 943 - 949

[30] Koizumi S, Kida M, Yamauchi H et al. Clinical implications of doubling time of gastrointestinal submucosal tumors. World J Gastroenterol 2016; 22: 10015-10023 